\title{
Equipos de salud mental de primera línea durante la pandemia de COVID-19
}

\section{Frontline mental health teams during the covid-19 pandemic}

José L. Jiménez-López*

Instituto Mexicano del Seguro Social, Centro Médico Nacional La Raza, Hospital de Especialidades "Dr. Antonio Fraga Mouret", Ciudad de México, México

Las investigaciones relacionadas con la infección por SARS-CoV-2 se han enfocado en los aspectos médicos de la enfermedad y poco se ha estudiado su impacto en la salud mental. De acuerdo con los Principios de Intervención Psicológica en Crisis, de China, los pacientes en aislamiento hospitalario por infección por SARS-CoV-2 confirmada se encuentran en el primer nivel de vulnerabilidad para problemas de salud mental. ${ }^{1}$ Estos pacientes son atendidos por los médicos de primera línea que no están capacitados para manejar aspectos emocionales; el personal de salud mental que debería atenderlos realiza evaluaciones en forma remota para evitar el contagio, porque la atención que proporciona se considera no esencial (existen reportes de que estos profesionales han sido redistribuidos para aumentar la capacidad de respuesta debido a la escasez de médicos). ${ }^{2-4}$

En todo el mundo se recomiendan los servicios de consulta remota en situaciones excepcionales como la actual pandemia, sin embargo, hay deficiencias que no pueden cubrirse como la obtención de datos de historia médica, psicometría y lenguaje corporal, por lo que no se puede reemplazar la consulta cara a cara. $^{2}$
Hasta ahora, no existen reportes internacionales sobre la creación de equipos de salud mental para la atención presencial de pacientes en aislamiento por COVID-19. En el Instituto Mexicano del Seguro Social se creó un equipo médico con ese objetivo. ${ }^{5}$

La evaluación presencial por psiquiatras y psicólogos o psicólogas de pacientes en aislamiento por COVID-19 puede aportar información más precisa sobre los motivos del malestar emocional y permitir intervenciones más efectivas; disminuir el malestar psicológico y tratar los trastornos mentales diagnosticados, lo cual podría tener repercusiones importantes en el pronóstico de la enfermedad dada la conocida influencia del estado anímico sobre el sistema inmunitario.

\section{Bibliografía}

1. National Health Commission of the People's Republic of China [Internet]. China: Principles of the emergency psychological crisis interventions for the new coronavirus pneumonia; 2020.

2. Jiang X, Deng L, Zhu Y, Ji H, Tao L, Liu L, et al. Psychological crisis intervention during the outbreak period of new coronavirus pneumonia from experience in Shanghai. Psychiatry Res. 2020;286:112903.

3. McNamara D. Neurologists dust off their stethoscopes, join COVID front lines [Internet]. Medscape; c2020.

4. Lieberman JA. NY psych residents describe 'tsunami' of COVID-19 patients [Internet]. Medscape; c2020.

5. Equipo de salud mental. COVID-19. Boletín Científico del Hospital de Especialidades "Dr. Antonio Fraga Mouret". 2020;1(2). 